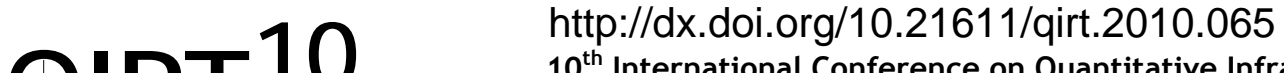 \\ $10^{\text {th }}$ International Conference on Quantitative InfraRed Thermography \\ July 27-30, 2010, Québec (Canada)
}

\section{Quantitative infrared wall inspection}

by T. Kisilewicz* ${ }^{\star}$ A. Wróbel**

\author{
${ }^{\star}$ CE Dept., Cracow University of Technology, Kraków, Poland, tkisilew@pk.edu.pl \\ ** MSaEE Dept., AGH University of Science and Technology, Kraków, Poland, alibel@agh.edu.pl
}

\begin{abstract}
Energy performance certification of an existing building should be based on a reliable analysis of the wall thermal characteristics. In the simplest case it may rely on the building plans, if they exist and are available. Since those plans may reflect designer's intention rather than the reality, it is suggested that the final reliable assessment should be based only on experimental testing, e.g. an infrared inspection [1]. A well insulated wall was tested in laboratory climatic chamber, initially under stationary and then under non-stationary conditions. Data gathered simultaneously by means of heat flow meter and infrared camera allowed to determine thermal characteristic of the wall in different ways. The results of the comparative measurements are presented in this paper.
\end{abstract}

\section{Introduction}

Infrared inspection of the existing buildings is very often aimed not only at answering the question whether a continuous thermal insulation is present, but also at determining the extent of expected thermal losses. Such an approach means turning from qualitative, as in the standard [1], to quantitative thermographic assessment of the building.

For this purpose, general conditions of the inspection assuring reliable and sufficient data acquisition should be specified. Subsequently, common criteria of the objective thermal image evaluation should be precisely stated. The main difficulty with field inspection of the building shell in real internal and external conditions, so called "in-situ" examination [2], is related to non-stationary boundary conditions. Due to continuous changes in the environmental temperatures, not only thermal conductivity but also specific heat and density of the materials significantly influence heat transfer through a building component. Instantaneous temperature distribution, recorded during a quick thermographic survey does not correspond with the stationary heat flow conditions and, usually, is not a good basis for reliable calculation of the overall heat transmission coefficient value.

The best way to overcome temperature and heat fluctuations in real conditions is to collect data for a sufficiently long period (respectively to component thermal capacity) and use mean values to calculate thermal resistance [2].

Influence of the non-stationary conditions on the results of inspection may be to some extent limited when the inspection is carried out during a carefully selected period with limited and repeating air temperature fluctuations.

Other factors having impact on success of the thermographic inspection, like solar radiation, precipitation and intensive air movement, relative humidity, distance, sky and environmental radiation etc., are relatively well researched and known by now.

\section{Experimental testing in the climatic chamber}

The testing facility of the Cracow Technical University (CUT), Figure 1, contains the warm and cold chambers, which allow investigating a sample of maximum size $1.9 \times 1.9 \mathrm{~m}$.

The wall tested in the climatic chamber consisted of two layers: the silica structural brickwork and insulation of 12 $\mathrm{cm}$ thick foamed polystyrene coated with $5 \mathrm{~mm}$ reinforced plaster, as in ETICS (External Thermal Insulation Compound System).

Temperatures of air and wall surfaces were simultaneously measured by means of the T-type thermocouples and thermal camera Therma CAM S60 PAL with $20^{\circ}$ lens, Figure 2. A sheet of paper mounted on the tripod within the camera field of view was used to allow air temperature observation. Thermal images were recorded at 30 minute intervals. Temperature measured on the thermal image was in ThermaCAM Reasearcher Pro2.8, averaged across selected areas, as shown in Figure 2 with accuracy $0.1 \mathrm{~K}$.

Heat flow density was measured on the warm surface of the wall by the heat flow transducer Ahlborn W 150-2 (50 $\mathrm{cm} \times 50 \mathrm{~cm}$ ) with calibration uncertainty of $5 \%$. 


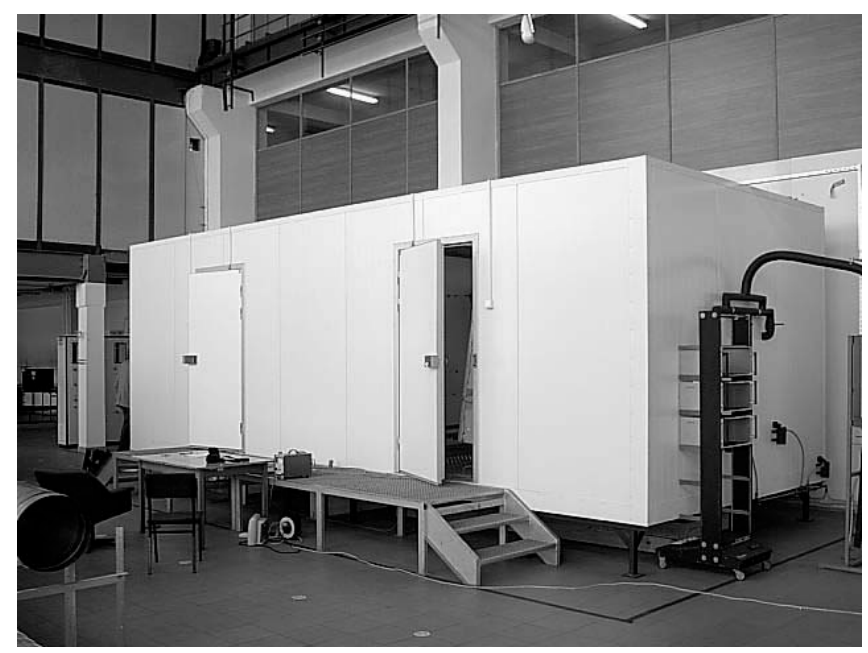

Fig. 1. Full scale climate chamber facility at Cracow University of Technology, cold chamber on the left, warm chamber on the right side.
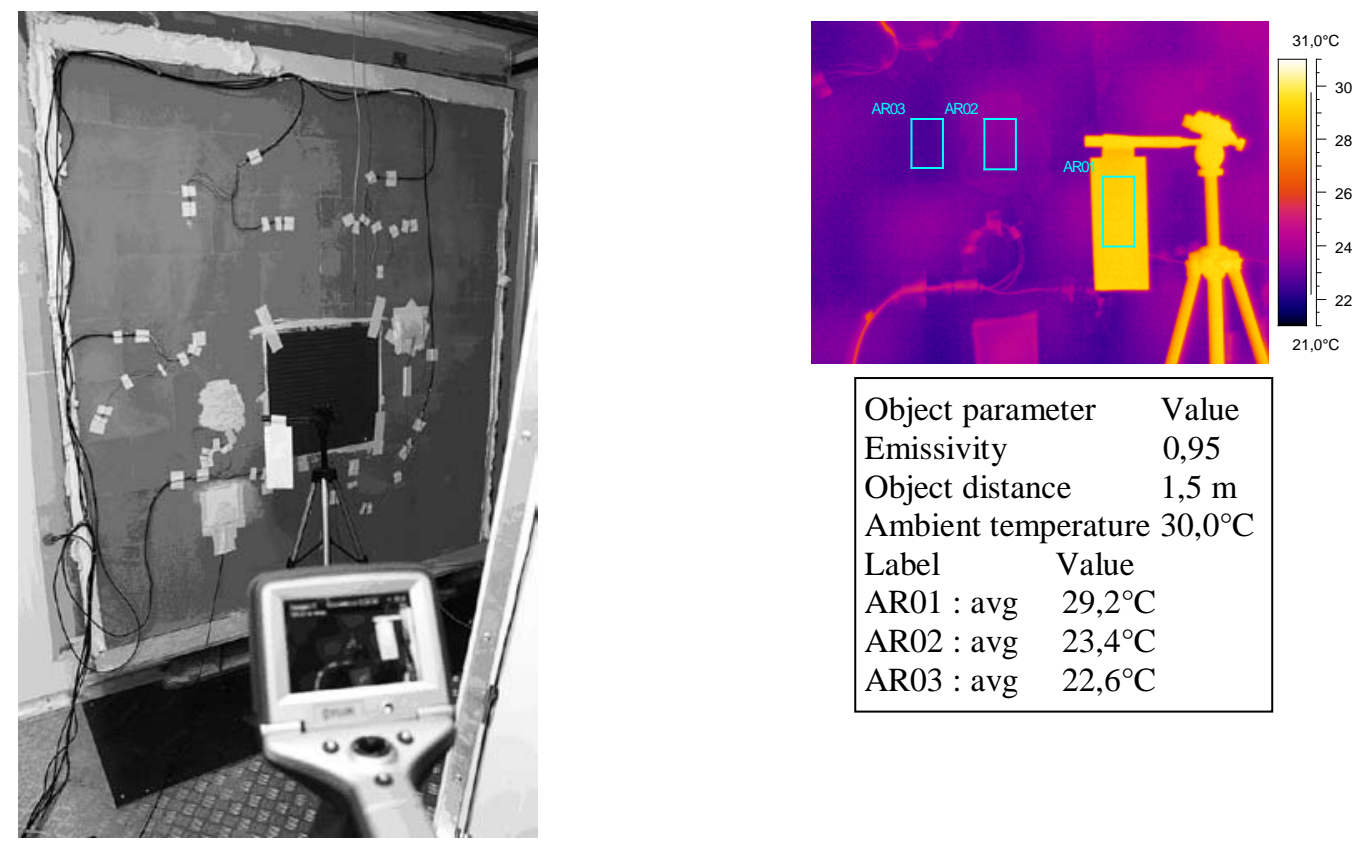

Object parameter Value

Emissivity $\quad 0,95$

Object distance $\quad 1,5 \mathrm{~m}$

Ambient temperature $30,0^{\circ} \mathrm{C}$

Label Value

AR01 : avg $29,2^{\circ} \mathrm{C}$

AR02 : avg $23,4^{\circ} \mathrm{C}$

AR03 : avg $22,6^{\circ} \mathrm{C}$

Fig. 2. Warm chamber of the testing facility. On the left: a view of the sample wall with the thermocouples, heat flow transducer and the thermal camera, on the right: thermal image of the sample, tripod with paper sheet and the areas selected for temperature averaging

Heat transfer at the interior surface of a wall is usually described by a Newton's boundary condition, Eq. (1):

$$
q_{s i}=h_{s i} \cdot\left(T_{i}-T_{s i}\right)
$$

where:

$\mathrm{h}_{\mathrm{si}}$ - heat transfer coefficient at the internal wall surface, $\mathrm{W} /\left(\mathrm{m}^{2} \mathrm{~K}\right)$

$\mathrm{T}_{\mathrm{i}}$ - ambient air temperature, ${ }^{\circ} \mathrm{C}$,

$\mathrm{T}_{\mathrm{si}}$ - internal surface temperature, ${ }^{\circ} \mathrm{C}$. 
A moderate forced convection was observed in the warm chamber. On the cold side, intensive forced convection conditions matched average exterior conditions as in the European standard EN 6946 [4]. Average velocity of the forced air flow in the warm chamber was ca. $0.3 \mathrm{~m} / \mathrm{s}$. Surface heat transfer coefficient $h_{\mathrm{si}}$, calculated according to the European standard EN 6946 [4] as the sum of forced convection and radiation coefficients, was equal to $10.33 \mathrm{~W} /\left(\mathrm{m}^{2} \cdot \mathrm{K}\right)$.

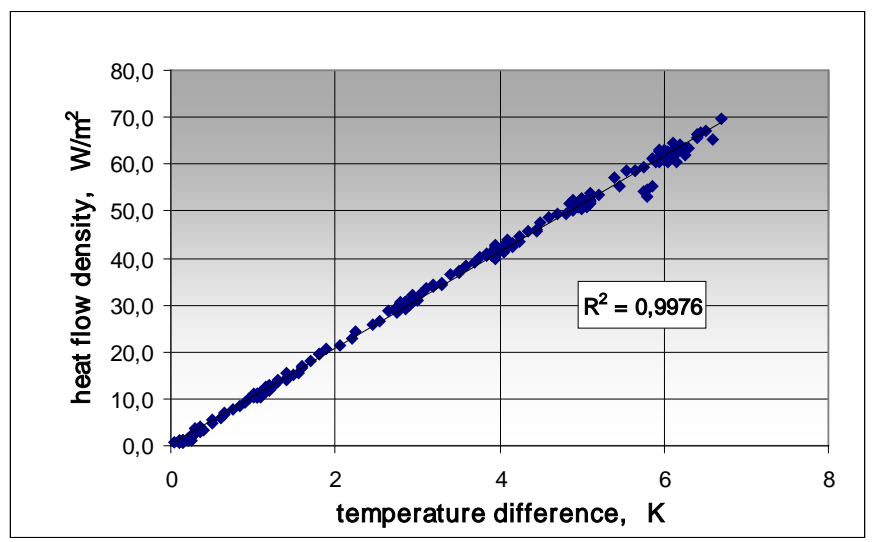

Fig. 3. Correlation of the measured heat flux density and recorded by the thermal camera temperature difference of air and wall surface in the warm chamber

Heat flux density data, measured by means of the transducer and recorded by the thermal camera temperature difference of air and wall surface in the warm chamber, are plotted in figure 3. A very strong correlation may be easily observed: correlation coefficient is greater than 0.99 with standard deviation of $0.45 \mathrm{~W} / \mathrm{m}^{2}$. It proves that the thermal camera measurement accuracy should be sufficient for quantitative assessment of the wall.

According to Eq. (1), the slope of the approximating line can be regarded as the surface heat transfer coefficient $h_{s i}$. In our case, the slope resulting from the measurements equal to $10,28 \mathrm{~W} /\left(\mathrm{m}^{2} \cdot \mathrm{K}\right)$ was practically the same as the above calculated value [5].

\section{Testing results}

The standard testing procedure in the climate chamber is usually based on stationary heat flow conditions, i.e. constant temperature in both chambers. Thermal resistance of the wall was measured in the stationary boundary conditions: $\mathrm{T}_{\mathrm{i}}=+30,20^{\circ} \mathrm{C}$ and $\mathrm{T}_{\mathrm{e}}=+2,05^{\circ} \mathrm{C}$. Thermal resistance of the insulated wall in these conditions $\mathrm{R}_{\lambda, \text { stat }}$ was equal to 2,68 $\mathrm{m}^{2} \cdot \mathrm{K} / \mathrm{W}$. It was regarded later as a point of reference.

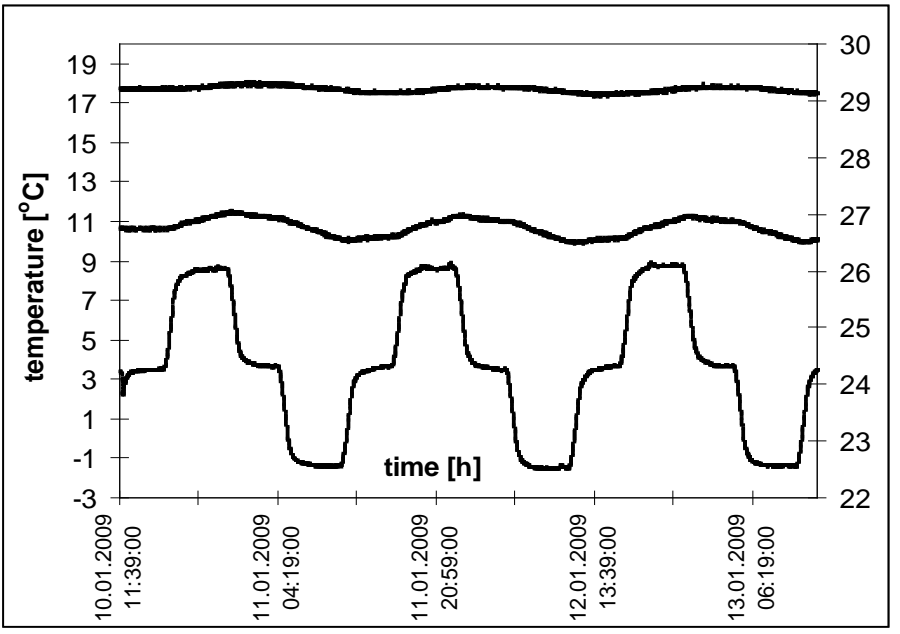

Fig. 4. Quasi-periodic temperature fluctuations in the cold chamber for ilnsulated silica brickwork: temperature fluctuations at the interior wall surface (upper curve and right axis), between insulation and brickwork (middle curve, right axis) and ambient air in the cold chamber (bottom curve and left axis). 
Subsequently non-stationary testing courses were started. The temperature control system of the chamber allows not only to maintain steady state conditions but also to follow the stepwise programmed temperature schedule. This feature of the testing facility was used to simulate non-stationary conditions in the cold chamber. A test that was designed to model sinusoidal ambient air temperature schedule is shown in figure 4. In fact, although the temperature controlling unit had been programmed to follow the stepwise procedure, due to the steering process logic and chamber thermal capacity, the cold air temperature course was partially smoothed. It was assumed that the periodic temperature oscillations were the closest match to real day and night temperature changes. Due to the quasi-sinusoidal ambient air temperature fluctuations, the mathematical solution of the heat transfer in harmonic boundary conditions was commonly used to determine the dynamic characteristics of the building walls as in the standard EN 13786 [3].

The diagrams in figure 4 (cold and warm surface temperature) show thermal response of the he sample wall to the stepwise programmed temperature controller. Maximum ambient air temperature was $+8^{\circ} \mathrm{C}$ and minimum $-2,5^{\circ} \mathrm{C}$. In order to show clearly the fluctuations of the well damped temperature wave, two different temperature ranges (and axes) were used in Figure 4. The cold surface temperature amplitude is $10.17 \mathrm{~K}$ with mean value of $2.64{ }^{\circ} \mathrm{C}$. The amplitude of the temperature fluctuations under the insulation is reduced to $0.58 \mathrm{~K}$ and the interior surface temperature amplitude is, at the same time, equal to $0.27 \mathrm{~K}$. The damping factor of the insulated wall was 0.027 .

A quasi-sinusoidal $24 \mathrm{~h}$ period was applied to simulate the random external climate conditions. Instantaneous heat flux densities measured by the heat flow transducer and calculated using the thermal images and Eq. (1) is shown in figure 5. Due to the high sensitivity of the heat flow sensor to the air turbulences and electrical noise data recorded by the camera show a close fit to the physical variability of the heat flow.

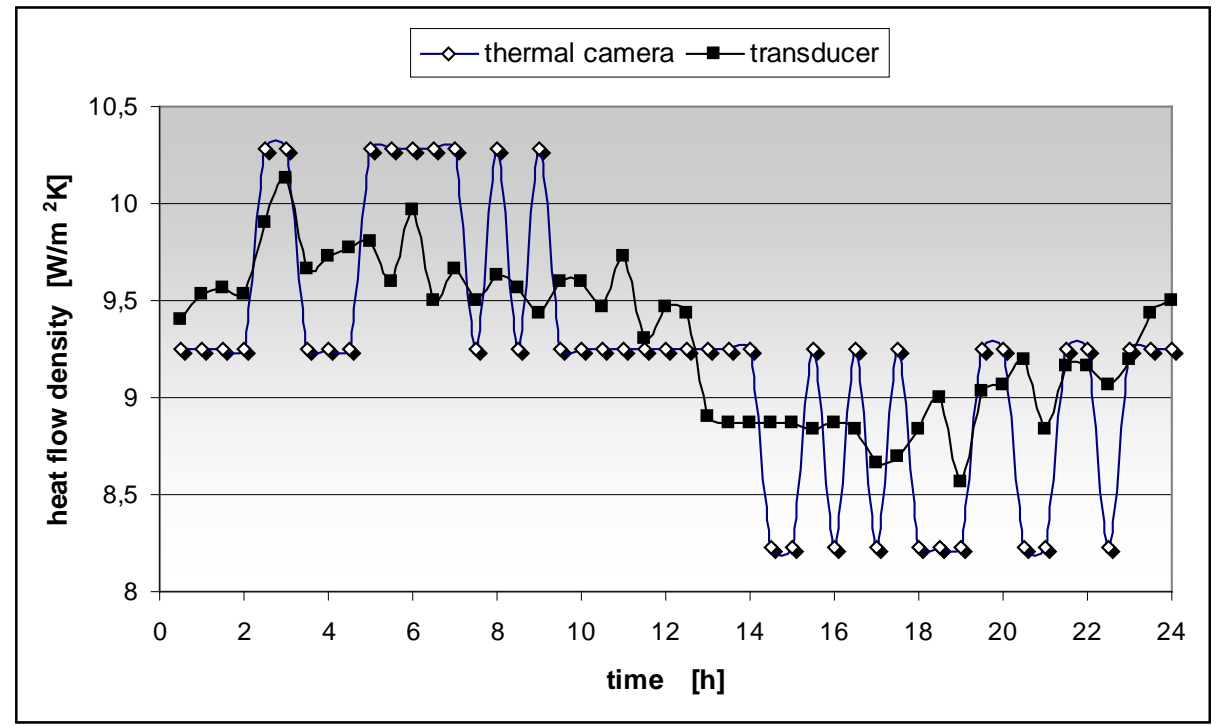

Fig. 5. Quasi-periodic temperature fluctuations. Heat flux at the internal wall surface measured by the heat flow transducer and thermal camera.

So called "in-situ" examination of the existing building shell is always connected with non-stationary boundary conditions. However, instantaneous temperature distribution, recorded during a quick thermographic survey (as it often happens in a common practice) is not a good basis for reliable calculation of the wall thermal resistance value. The possible results of the instantaneous measurements are shown in figure 6 as the separate points. Under the non-stationary conditions such a quick, momentary survey could become a source of the completely false results. The thermal resistance of the wall measured in this way would be in the range of 2.07 to $3.84 \mathrm{~m}^{2} \mathrm{~K} / \mathrm{W}$ while the proper value is $2.68 \mathrm{~m}^{2} \mathrm{~K} / \mathrm{W}$.

In real circumstances, the best way to overcome temperature and heat flow fluctuations in real conditions is to collect data for a sufficiently long period and use mean values to calculate the thermal resistance. According to the draft standard prEN 12494 [2], an analysis shall be carried out over a period which is an integer multiple of 24 hours. In our case data recorded by the infrared camera during the regular quasi-sinusoidal 24 and $48 \mathrm{~h}$ periods were used to calculate wall thermal resistance. 
The mean heat flux $q_{\text {mean,si }}$ was calculated as the product of the surface heat transfer coefficient and mean temperature drop at the internal surface, Eq. (2):

$$
q_{\text {mean,si }}=h_{\text {si }} \cdot\left(T_{i}-T_{\text {si }}\right)_{\text {mean }}
$$

where:

index "mean" - is 24 or $48 \mathrm{~h}$ mean value of the heat flux and surface temperature drop

The thermal resistance of the wall $\mathrm{R}_{\lambda}$ was calculated as a ratio of the mean temperature difference of the interior and exteriorl wall surfaces and the above calculated heat flux, Eq. (3):

$$
R_{\lambda}=\frac{\left(T_{\text {si }}-T_{\text {se }}\right)_{\text {mean }}}{q_{\text {mean }, s i}}
$$

where:

$\mathrm{T}_{\mathrm{se}}-$ momentary exterior wall surface temperature, ${ }^{\circ} \mathrm{C}$.

$\mathrm{R}_{\lambda, 24}$ as calculated by Eq. (3) was equal to $2,86 \mathrm{~m}^{2} \mathrm{~K} / \mathrm{W}$, i.e. $6,7 \%$ greater than that measured under stationary conditions. The period extended to 48 hours improved accuracy of the calculations: $R_{\lambda, 48}$ was equal to $2,73 \mathrm{~m}^{2} \mathrm{~K} / \mathrm{W}$, i.e. $1,7 \%$ greater than that measured under stationary conditions. It all means that the infrared imaging may be successfully used for quantitative assessment of the building external shell. Any increased uncertainty of the measurements is likely a result of the chosen method and boundary conditions rather than a fault of the tool (camera).

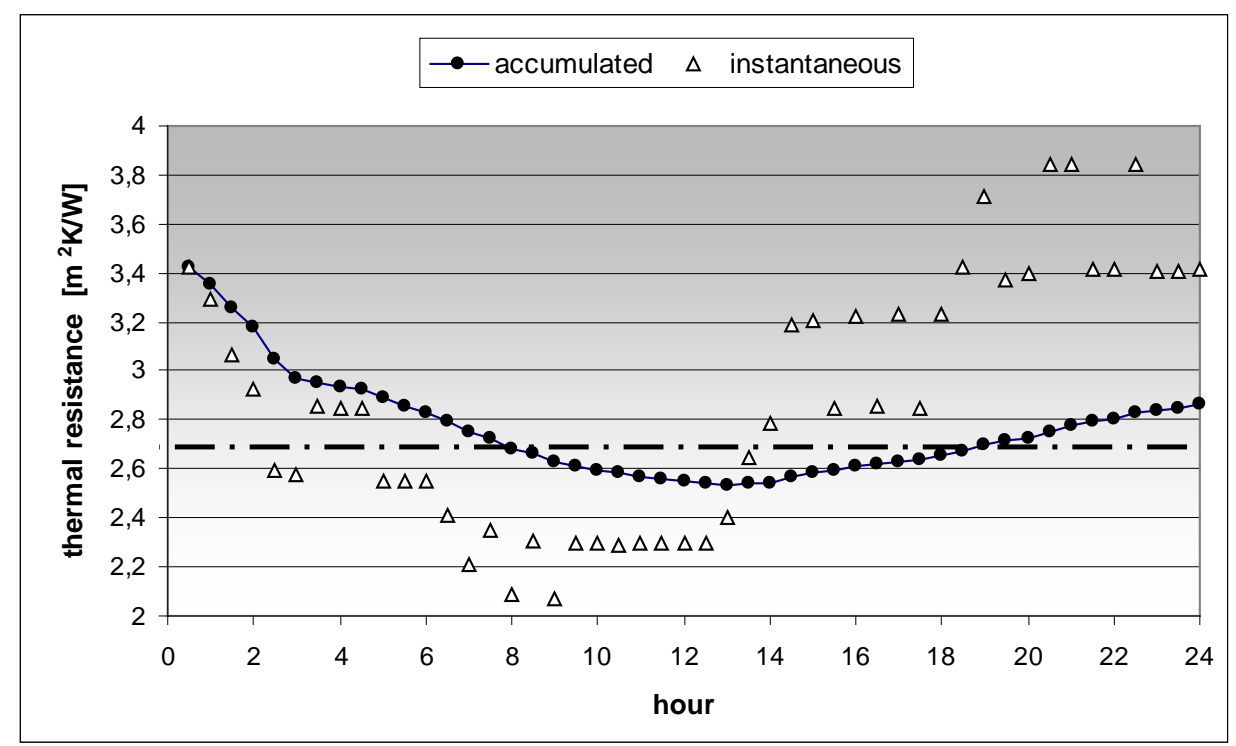

Fig. 6. Quasi-periodic temperature fluctuations in the cold chamber. Instantaneous and accumulated for $24 \mathrm{~h}$ thermal resistance of the wall. Black horizontal line refers to the stationary conditions value.

As it was mentioned above, the thermal analysis under real weather non-stationary conditions should be carried out over a period which is an integer multiple of 24 hours [2] i.e. a full period of the harmonic temperature wave. Special importance of this condition is shown in figures 6 and 7. A curve connecting black dots represents so called "accumulated" thermal resistance value. Every point of this curve was calculated as per Eq. (3), but the number of the applied averaged data was gradually increasing. In case of the full two cycles (48 h) i.e. "sufficiently long period of calculations" the thermal resistance of the tested wall would become about equal to that calculated under stationary conditions. 


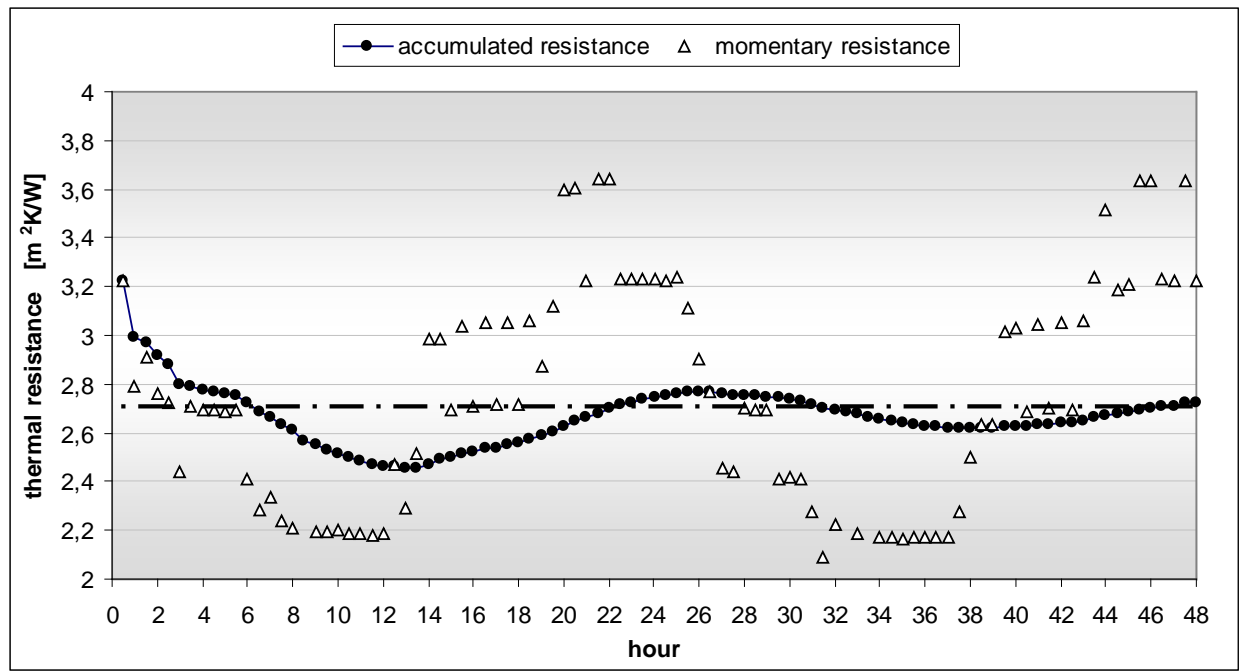

Fig. 7. Quasi-periodic temperature fluctuations in the cold chamber. Instantaneous and accumulated for $48 \mathrm{~h}$ thermal resistance of the wall. Black horizontal line refers to the stationary conditions value.

\section{Conclusions} justified.

It was shown that a transfer from qualitative to quantitative thermographic assessment of the building shell quality is

If the surface heat transfer value is correctly evaluated (e.g. according to the ISO standard procedures), the thermal camera measurement accuracy shall be sufficient for quantitative assessment of the wall.

In real climatic conditions sufficiently long period of data averaging and integer multiple of the $24 \mathrm{~h}$ period, i.e. the typical measures applied in testing of the building components under non-stationary conditions, would allow to achieve good results also in case of the infrared thermal inspection.

\section{Acknowledgements}

This work was carried out within the framework of the research grant: NN526119133 financed by the Polish Ministry of Science and Higher Education from 2007 to 2010.

\section{REFERENCES}

[1] EN 13187 "Thermal performance of buildings - Qualitative detection of thermal irregularities in building envelopes Infrared method".

[2] prEN 12494 "Building components and elements - In-situ measurement of the surface-to-surface thermal resistance"

[3] EN 13786, 2007. Thermal performance of building components - Dynamic thermal characteristics - Calculation methods.

[4] EN ISO 6946 "Building components and building element - Thermal resistance and thermal transmittance Calculation method".

[5] Kisilewicz T., Wróbel A. 2008. "Detection of thermal bridges - aims, possibilities and conditions". QUIRT 2008 Proceedings - 9-th International Conference on Quantitative Infrared Thermography, July 2-5, Krakow (Poland), p. 227-232, 2008.

[6] Kisilewicz T., "Thermal resistance of a wall determined in non-stationary boundary conditions", paper accepted for the I Central European Symposium on Building Physics, September 13-15, Krakow (Poland), 2010 